\title{
COMPONENT ORBITS UNDER POINTWISE RECURRENT HOMEOMORPHISMS
}

\author{
MARIANO GARCIA
}

1. Introduction. The notion of a component orbit is due to G. E. Schweigert, ${ }^{1}$ and is closely associated with two theorems established by D. W. Hall and G. E. Schweigert, ${ }^{2}$ but so far has been considered only in connection with pointwise periodic homeomorphisms. It is the purpose of this paper to consider an extended concept of this notion and, by applying methods analogous to those used by G. T. Whyburn ${ }^{3}$ in obtaining the results of Hall and Schweigert, exhibit certain theorems that contain these results as special cases.

2. Preliminary definitions and remarks. Throughout this paper, $X$ will denote a separable metric space. Let $f(X)=X$ be a homeomorphism defined on $X$ and let $x \in X$.

The point $x$ is recurrent under $f$ provided that given any neighborhood $U$ of $x$, there exists a positive integer $n$ such that $f^{n}(x) \in U$.

The mapping $f$ is pointwise recurrent on the set $A \subset X$ provided each point of $A$ is recurrent under $f$. It should be noted that pointwise recurrence, as defined here, is equivalent to pointwise almost periodicity as used by Ayres, Whyburn, and others.

A non-empty invariant set $L$ whose components can be ordered in a sequence

$$
\cdots, A_{-2}, A_{-1}, A_{0}, A_{1}, A_{2}, \cdots
$$

such that

$$
f\left(A_{i}\right)=A_{i+1}, \quad i=0, \pm 1, \pm 2, \cdots,
$$

will be termed a component orbit.

A component orbit one of whose components is invariant under a positive power of $f$ will be termed a periodic component orbit.4

Presented to the Society, February 26, 1944; received by the editors December 29, 1943. The author wishes to thank Professors G. A. Hedlund and G. T. Whyburn for their genial interest and encouragement in connection with the preparation of this paper.

${ }^{1}$ A note on the limit of orbits, Bull. Amer. Math. Soc. vol. 46 (1940) pp. 963-969.

2 Properties of invariant sets under pointwise periodic homeomorphisms, Duke Math. J. vol. 4 (1938) pp. 719-724.

3 Analytic Topology, Amer. Math. Soc. Colloquium Publications vol. 28 (1942) pp. 259-261. We shall refer to this book hereafter as ATW.

4 Schweigert's component orbits become periodic component orbits under this definition. 
By the set-orbit of a set $A \subset X$ we mean

$$
\sum_{-\infty}^{+\infty} f^{i}(A)
$$

and a set-orbit is non-wandering ${ }^{5}$ if there exists a positive integer $N$ such that $A \cdot f^{N}(A) \neq 0$.

It follows without difficulty that a component orbit is the set-orbit of a connected set, and conversely, the set-orbit of a connected set is a component orbit. Also, a periodic component orbit is the non-wandering set-orbit of a connected set, and conversely, the non-wandering set-orbit of a connected set is a periodic component orbit. Also, if a component orbit contains a periodic component orbit, it is a periodic component orbit, and in a compact space $X$, a component orbit is periodic if it is closed. Finally we remark that the orbit of a point $x$

$$
\sum_{-\infty}^{+\infty} f^{i}(x)
$$

is a component orbit, and if $x$ is periodic, its orbit is a periodic component orbit.

3. The main results. We now prove the principal theorems.

THEOREM I. Let $f(X)=X$ be a homeomorphism on a compact space $X$. If $G_{1}, G_{2}, \cdots$ is a sequence of component orbits whose limit inferior contains a periodic component orbit $Q$, and if both $f$ and $f^{-1}$ are pointwise recurrent on $\lim \sup G_{i}-Q$, then $\lim \sup G_{i}$ is a periodic component orbit.

Proof. Let $Q_{0}$ be a component of $Q$ and let $K_{0}$ be the component of $L=\lim \sup G_{i}$ which contains $Q_{0}$. Since $L$ is invariant, the image of $K_{0}$ under any power of $f$ is a component of $L$. Let $f\left(K_{0}\right)=K_{1}, f\left(K_{1}\right)=K_{2}, \cdots, f\left(K_{r-1}\right)=K_{r}, f\left(K_{r}\right)=K_{0}$, so that

$$
K=\sum_{0}^{r} K_{i}
$$

is a periodic component orbit which contains $Q$. We shall show $K=L$.

Suppose this is not true. Then we can find ${ }^{6}$ an open set $V$ containing $K$, whose boundary $F(V)$ does not intersect $L$ and such that $L$ is not contained wholly in $V$.

B In this connection see G. D. Birkhoff and P. A. Smith, Structure analysis of sur. face transformations, J. Math. Pures Appl. vol. 7 (1928) p. 350.

- ATW, Theorem (9.3), p. 15. The proof of Theorem I follows closely the proof of Theorem (6.5), p. 259. 
Select a point $x_{i}$ from each $G_{i}$ such that $x_{i} \rightarrow x \in Q_{0}$. Infinitely many $G_{i}$ intersect $X-\bar{V}$ and thus we may assume that for each $i$ there exists a point $y_{i} \in G_{i}$ such that $y_{i} \in X-\bar{V}$. Let $C_{x_{i}}$ be the component of $G_{i}$ containing $x_{i}$. Since $x \in \lim \inf C_{x_{i}}, \lim \sup C_{x_{i}}$ is connected ${ }^{7}$ and hence is contained in $K_{0}$. Also, ${ }^{8}$ for almost all $i$, and therefore we may assume for all $i, C_{x_{i}} \subset V$. Each $C_{x_{i}}$ contains a point $z_{i}$ of the orbit of $y_{i}$. A subsequence of $\left\{z_{i}\right\}$, which we may suppose is the whole sequence, converges to a point $z \in K_{0}$.

There exists a subsequence $\left\{z_{i_{j}}\right\}$ of $\left\{z_{i}\right\}$ such that for each $i_{j}, y_{i_{j}}$ is a positive power of $z_{i_{j}}$ or for each $i_{j}, y_{i_{j}}$ is a negative power of $z_{i_{j}}$. We shall assume that this sequence is $\left\{z_{i}\right\}$ and that $y_{i}$ is a negative power of $z_{i}$ for each $i$, since the proof for the case where $y_{i}$ is a positive power of $z_{i}$ for each $i$ is perfectly analogous.

Now, let $b_{i}$ be the first point in the sequence

$$
z_{i}, f^{-1}\left(z_{i}\right), f^{-2}\left(z_{i}\right), \cdots
$$

which is in $X-V$. A subsequence of $\left\{b_{i}\right\}$, which again we may suppose is the whole sequence, converges to a point $b \in L$. Furthermore, $b \notin \bar{V}$, since $L \cdot F(V)=0$.

For each $i, f\left(b_{i}\right)$ is in $V$ and consequently

$$
f\left(b_{i}\right) \rightarrow f(b) \in V \cdot(L-K),
$$

since $L-K$ is invariant.

Now, there exists an integer $N_{1}$ such that

$$
f\left(b_{i}\right) \neq z_{i} \quad \text { for } i>N_{1} .
$$

This is true because otherwise we should have

and therefore

$$
f\left(b_{i}\right) \rightarrow z
$$

$$
b_{i} \rightarrow f^{-1}(z) \neq b .
$$

Thus the points $f\left(b_{i}\right), i>N_{1}$, are negative powers of $z_{i}$ which are in $V$, and therefore the points $f^{2}\left(b_{i}\right), i>N_{1}$, are in $V$. Also,

$$
f^{2}\left(b_{i}\right) \rightarrow f^{2}(b) \in V \cdot(L-K) .
$$

But again, there exists an integer $N_{2}>N_{1}$ such that

$$
f^{2}\left(b_{i}\right) \neq z_{i} \quad \text { for } i>N_{2} \text {. }
$$

The points $f^{8}\left(b_{i}\right), i>N_{2}$, are then in $V$ and

\footnotetext{
ATW, Theorem (9.1), pp. 14-15.
}

8 ATW, Theorem (7.2), p. 12. 


$$
f^{3}\left(b_{i}\right) \rightarrow f^{3}(b) \in V \cdot(L-K) .
$$

But we can find an integer $N_{3}>N_{2}$ such that

$$
f^{3}\left(b_{i}\right) \neq z_{i} \quad \text { for } i>N_{3},
$$

and continuing in this manner we obtain

$$
f^{n}(b) \in V, \quad n=1,2,3, \cdots .
$$

This contradicts the recurrence of the point $b$ under $f$, since $b \notin \bar{V}$ and therefore we can find a neighborhood $R$ of $b$ such that

$$
f^{n}(b) \cdot R=0, \quad n=1,2,3, \cdots .
$$

Had we considered a sequence $\left\{z_{i}\right\}$ for which $y_{i}$ is a positive power of $z_{i}$ for each $i$, we would have obtained a contradiction to the recurrence under $f^{-1}$ of some point of $\lim \sup G_{i}-Q$.

THEOREM II. Let $f(X)=X$ be a homeomorphism on a compact space $X$. If $G_{1}, G_{2}, \ldots$ is a sequence of component orbits whose limit inferior contains a periodic component orbit $Q$ and if $f$ is pointwise recurrent on $\lim \sup G_{i}+\sum_{i=1}^{+\infty} G_{i}$, then $\lim \sup G_{i}$ is a periodic component orbit.

Proof. This follows from the proof of the preceding theorem. Let $O_{i}$ be the orbit determined by $y_{i}$ and $z_{i}$ in the proof of Theorem I. There exists a neighborhood $U_{i}$ of $z_{i}$, of diameter less than $1 / i$, such that $U_{i} \subset V$ and, due to the recurrence of $z_{i}$ under $f$, corresponding to $U_{i}$ we can find a point $r_{i} \in U_{i}$ such that $r_{i}$ is a positive power of $y_{i}$. Let $b_{i}$ be the first point in the sequence

$$
r_{i}, f^{-1}\left(r_{i}\right), f^{-2}\left(r_{i}\right), \cdots
$$

which is in $X-V$. Since $r_{i} \rightarrow z$, we can proceed as in the proof of Theorem I to show that the point $b$, to which we may assume $\left\{b_{i}\right\}$ converges, is not recurrent under $f$.

From the previous proofs we immediately have the following corollary.

CoRollary 1. Under the hypotheses of Theorem I or of Theorem II, the number of components of $\lim$ sup $G_{i}$ can not exceed the number of components of $Q$. Thus, if lim inf $G_{i}$ contains an invariant connected set (in particular, if it contains a fixed point), then $\lim \sup G_{i}$ is connected.

CoROLLARY 2. Let $f(X)=X$ be a homeomorphism on a compact space $X$. Let $G$ be a component orbit in $X$. If $f$ is pointwise recurrent on $\bar{G}$ and $\bar{G}$ contains a periodic component orbit $Q$, then $\bar{G}$ is a periodic component orbit and the number of its components does not exceed the number of components of $Q$. 
Proof. This is a direct consequence of Theorem II and Corollary 1 , for we need only take $G_{i}=G$ for all $i$. Then $\lim G_{i}=\bar{G}$ and the conclusion follows.

Corollary 3. Any pointwise periodic continuous mapping $g$ of a compact totally disconnected space $X$ into itself has equicontinuous powers. ${ }^{9}$

Proof. It is a well known fact ${ }^{10}$ that under the above hypotheses $g$ is a homeomorphism of $X$ onto itself. Let us show that the orbit decomposition given by $g$ is continuous. ${ }^{11}$

Let $\left(O_{n}\right)$ be a sequence of orbits of $X$ such that $x \in \lim \inf \left(O_{n}\right)$. Then, letting $O_{x}$ be the orbit of $x$,

$$
O_{x} \subset \lim \inf \left(O_{n}\right) \subset \lim \sup \left(O_{n}\right) .
$$

Now, by Theorem I, lim sup $\left(O_{n}\right)$ is a periodic component orbit and, since $X$ is totally disconnected, lim sup $\left(O_{n}\right)$ is an orbit.

Thus, $\lim \left(O_{n}\right)=O_{x}$ and therefore the orbit decomposition is continuous, from which it follows that $f$ has equicontinuous powers. ${ }^{12}$

THEOREM III. Let $f(X)=X$ be a homeomorphism on a compact space $X$. If $L=L_{1}+L_{2}$ is a separation of a non-empty, closed, invariant set $L$ in $X$ such that $f$ is pointwise recurrent on $L$ and, for $i=1$ or for $i=2$, $L_{i}$ has the property that the closure of the orbit of each of its points is a periodic component orbit, ${ }^{13}$ then there exists a positive integer $N$ such that

$$
f^{N}\left(L_{i}\right)=L_{i}, \quad i=1,2 .
$$

Proof. It is no restriction to assume that $L_{1}$ has the indicated property: $x \in L_{1}$ implies that the closure of the orbit of $x$ is a periodic component orbit.

Since $f$ is pointwise recurrent ${ }^{14}$ on $L$, if the theorem is false, for each $n$ there exists an $x_{n} \in L_{1}$ such that $f^{n !}\left(x_{n}\right) \in L_{2}$.

Choose a subsequence $\left\{x_{n_{i}}\right\}$ of $\left\{x_{n}\right\}$ converging to a point $x \in L_{1}$. Let $O$ be the orbit of $x$. By hypothesis, 252.

' For the meaning of the terms involved in this corollary, see ATW, pp. 34, 239,

${ }^{10}$ ATW, Theorem (1.1) and Corollary (1.11), p. 240.

11 ATW, pp. 129-130.

12 ATW, Theorem (6.4), p. 257. See also Corollary (6.42), p. 258.

${ }^{13}$ We could substitute "contains a periodic component orbit" for the phrase "is a component orbit," but in virtue of Corollary 2 this does not matter.

14 In connection with the phrase that follows, see ATW, Theorem (4.12), p. 247, keeping in mind that "pointwise almost periodic," as used there, is "pointwise recurrent" in our notation. 


$$
\bar{O}=A_{0}+A_{1}+\cdots+A_{k},
$$

where $A_{0}$ is a component of $\bar{O}$ and

$$
f\left(A_{i}\right)=A_{i+1}, \quad i=0,1, \cdots, k-1,
$$

and $f\left(A_{k}\right)=A_{0}$. We may also assume that $A_{r} \neq A_{\text {s }}$ for $r \neq s, r$ and $s$ being any integers, $0 \leqq r, s \leqq k$.

Then $x \in A_{j}$, where $j$ is an integer such that $0 \leqq j \leqq k$.

Define $g(y)=f^{k}(y), y \in L$. By a result of W. H. Gottschalk, ${ }^{15} g$ is pointwise recurrent on $L$.

For each $n_{i}$, let $G_{n_{i}}$ be the orbit under $g$ of $x_{n_{i}}$. Now,

$$
x \in \lim \inf G_{n_{i}} \text {. }
$$

Furthermore, since

$$
A_{j}=\frac{\sum_{i=-\infty}^{+\infty} f^{k i}(x) \subset \lim \inf G_{n_{i}},}{\sum_{i=-\infty}^{+\infty} f^{k i}(x) \subset \lim \inf G_{n_{i} .} .}
$$

But now $g(L)=L$ is a homeomorphism on the compact set $L$ and $\left\{G_{n_{i}}\right\}$ is a sequence of orbits whose limit inferior contains the connected set $A_{j}$. Also, $A_{j}$ is invariant under $g$ and $g$ is pointwise recurrent on $L$. Thus, by Theorem II, $\lim \sup G_{n_{i}}$ is connected and consequently

$$
\lim \sup G_{n_{i}} \subset L_{1},
$$

since $x \in L_{1}$

But for each $i$,

$$
f^{n_{i 1}}\left(x_{n_{i}}\right) \in L_{2}
$$

and for $n_{i}>k$,

$$
f^{n_{i !}}\left(x_{n_{i}}\right)=g^{n_{i} ! / k}\left(x_{n_{i}}\right) .
$$

Thus it follows that $G_{n_{i}} \cdot L_{2} \neq 0$ for $n_{i}>k$. This gives

$$
L_{2} \cdot \lim \sup G_{n_{i}} \neq 0,
$$

which contradicts lim sup $G_{n_{i}} \subset L_{1}$.

4. Conclusion. The main results that Hall and Schweigert obtained relative to component orbits can be stated in the following way: ${ }^{16}$

15 Powers of homeomorphisms with almost periodic properties, Bull. Amer. Math. Soc. vol. 50 (1944) Corollary 1, p. 223. This result is very useful here.

${ }^{16}$ See footnote 3 . 
THEOREM 1. Let $f(X)=X$ be a pointwise periodic homeomorphism on a compact space $X$. Then if $G_{1}, G_{2}, \ldots$ is a convergent sequence of periodic component orbits in $X$, the limit set $L$ is a periodic component orbit.

TheOREM 2. Let $X$ be compact and suppose $f(X)=X$ is pointwise periodic. Any non-empty disconnected invariant closed set $L$ in $X$ has the property that for every separation

$$
L=L_{1}+L_{2}
$$

there exists an integer $N$ such that

$$
f^{N}\left(L_{i}\right)=L_{i}, \quad i=1,2 .
$$

Theorem 1 follows immediately from either of Theorems I and II of this paper, for since $f$ is pointwise periodic on $X, f$ and $f^{-1}$ are pointwise recurrent on $X$. Also, since $X$ is compact, any set which is the limit of a convergent sequence of component orbits is non-empty, and therefore contains a periodic orbit, which clearly is a periodic component orbit. Thus, the hypotheses of Theorems I and II are satisfied and hence $\lim G_{i}$ is a periodic component orbit. In this connection it may be remarked that a homeomorphism $f(X)=X$ is pointwise periodic if and only if every component orbit in $X$ is a periodic component orbit, where $X$ is not necessarily compact.

That Theorem $I$ is considerably more general than Theorem 1 is evident in more than one sense. Referring to the notation employed in Theorem I, examples satisfying the hypotheses of the theorem can be constructed for which $f$ is not pointwise recurrent on $Q$ and such that $f$ is not pointwise periodic on $\lim \sup G_{i}-Q$.

Theorem 2, which incidentally may be used to prove Corollary 3 of this paper, is a direct consequence of Theorem III. For if $f$ is pointwise periodic on $X$, the closure of any orbit in $X$ is a periodic component orbit; namely, the orbit itself.

Here again Theorem III is of more generality than Theorem 2. Using the notation of Theorem III, examples fulfilling the conditions of the theorem can be given for which there exist points in $L_{2}$, say, the closures of whose orbits are not even component orbits, and such that $f$ is not pointwise periodic on $L_{1}$ nor on $L_{2}$.

UNIVERSITY OF VIRGINIA 